\title{
Vibro-thermography of debonding defects in composite plates based on viscoelasticity heat
}

\author{
by Xingwang Guo* and Liang Zhu*
}

* Beihang University, School of Mechanical Engineering and Automation, 37 Xueyuan Rd. Haidian District, 100191, Beijing, China, xingwangguo@buaa.edu.cn, zhuliangfv@163.com

\section{Abstract}

The vibrothermography of disbonding defects in composite plates by using viscoelastic heating was studied. The influence of excitation frequency on the temperature increase over defects was analyzed using the thermomechanical coupled field modelling. The results show that the viscoelastic loss of the nonmetal material is the leading factor of the local temperature increase over defects; a defect corresponds to a so called higher mode characteristic frequency which can act as the optimal excitation frequency for this defect; the higher mode characteristic frequency is between the two LDR frequencies corresponding to the simple supported boundary and the clamped boundary respectively.

\section{Introduction}

Vibro-thermography (VT) has been paid more attention in recent years due to its high efficiency, good capability of detecting kissing defects and the feature of the defect selective dark field method. The principle of VT and the factors that govern the mechanical energy turning into heat during vibration are researched from different views by many teams all over the world, the most common views are that the heat generation in defects during VT comes from friction, thermoelasticity and viscoelasticity thermal effect $[1,2,3]$. The friction heat of cracks in metal had been studied thoroughly $[2,4$, 5]. The viscoelastic heating has been observed in polymer composite, carbon fibre reinforced composite [1] and viscous material-filled synthetic defects $[3,6]$, however, there is still a lack of theoretical model of the physics governing the heat generation process in vibrating, and some fundamental questions have remained unanswered, such as the influence of excitation on the temperature increase over defects, whether or not an optimal excitation frequency exists. The recent development of VT is the concept of local defect resonance (LDR), which indicates that using the LDR frequency as the excitation frequency can pump energy directly into the defect areas [7,8], thus achieving a better thermal effect.

In this paper, a thermo-mechanical coupled field model is established to study the VT of debonding defects in composite plates, focusing on the physical mechanism of heat generation based on viscoelasticity heat, and the influence of excitation frequency on the thermal effect over defects. The results of experiment and numerical simulation using finite element method are compared. A so called higher mode characteristic frequency is presented to act as the optimal excitation frequency a corresponding defect. The higher mode characteristic frequencies are calculated via the modal analysis of the whole sample. The temperature increases of the defects using the LDR frequencies and the higher mode characteristic frequencies as the excitation frequencies are compared by numerical simulation.

\section{Theoretical basis}

The analysis of vibration is based on the classical vibration theory of plates. For a plate under a transverse base excitation $W_{\mathrm{b}}(t)$ as shown in Fig.1, the differential equation of motion is given as Eq.(1):

$$
D \nabla w^{4}+\rho h \frac{\partial^{2} w}{\partial^{2} t}=-\rho h \ddot{w}_{\mathrm{b}}(t), \quad D=\frac{E h^{3}}{12\left(1-\mu^{2}\right)}
$$

where $w(x, y, t)$ is the relative displacement of the middle surface of the plate to the base, $\rho$ is the density, $D$ is the flexural rigidity, $E$ is Young's modulus, $\mu$ is Poisson's ratio, $h$ is the thickness of the plate.

The boundary condition at $y=0$ is given as Eq.(2). The boundary conditions at $y=b, x=0$ and $x=a$ are similar to Eq.(2), and they are omitted.

$$
\begin{aligned}
& \left(\frac{\partial^{2} w}{\partial y^{2}}+\mu \frac{\partial^{2} w}{\partial x^{2}}\right)_{y=0}=0 \\
& {\left[\frac{\partial^{3} w}{\partial y^{3}}+(2-\mu) \frac{\partial^{3} w}{\partial x^{2} \partial y}\right]_{y=0}=0}
\end{aligned}
$$




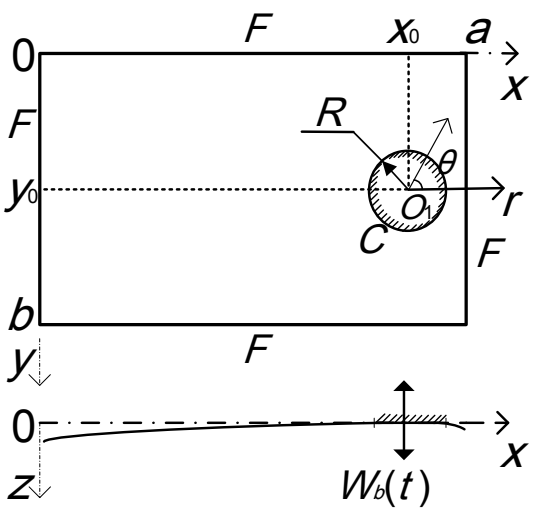

Fig.1. Drawing of a plate under the clamped base excitation, $F$ represents the free boundary, $C$ represents the clamped boundary

The boundary conditions at the circular border of the clamped zone can be described in the local cylindrical coordinate as:

$$
(w)_{r=R}=0,\left(\frac{\partial w}{\partial r}\right)_{r=R}=0
$$

For the transient conduction of heat in solids with internal heat source, the temperature obeys Eq.(3),

$$
k_{x} \frac{\partial^{2} T}{\partial x^{2}}+k_{y} \frac{\partial^{2} T}{\partial y^{2}}+k_{z} \frac{\partial^{2} T}{\partial z^{2}}+q=\rho c \frac{\partial T}{\partial t}
$$

where $T(x, y, z, t)$ is the temperature relative to ambient, $k_{i}(i=x, y, z)$ is the thermal conductivity in the $i$ direction, and it has $k_{x}=k_{y}=k_{z}=k$ for a isotropic plate, $c$ is the specific heat, $q(x, y, z, t)$ is the volumetric energy generation at the location $(x, y, z)$.

Making the assumption that the temperature varies much slower than the mechanical loading, then during the steady vibration phase, $q$ can be estimated as the time-averaged volumetric energy dissipated over one cycle of harmonic loading, as given by Eq.(5) [9],

$$
q=\eta \omega v_{0}=\frac{\eta \omega E z^{2}}{2\left(1-\mu^{2}\right)}\left[\left(\frac{\partial^{2} w}{\partial x^{2}}\right)^{2}+\left(\frac{\partial^{2} w}{\partial y^{2}}\right)^{2}+2 \mu \frac{\partial^{2} w \partial^{2} w}{\partial x^{2} \partial y^{2}}+2(1-\mu)\left(\frac{\partial^{2} w}{\partial x \partial y}\right)^{2}\right]
$$

where $\eta$ is the loss factor, $\omega$ is the angular frequency of excitation, $v_{0}$ is the strain energy density, $w=w(x, y, t)$ is the same as in Eq.(1).

Eq.(4) can be solved when it is combined with the necessary boundary and initial conditions that is omitted here.

\section{Experiment}

An ultrasonic IR thermographic system developed by Beihang University was used. The system includes an IR camera, an ultrasonic device and a computer with specialized software for image acquisition and processing. The ultrasonic device is equipped with three sets of ultrasonic piezoelectric transducers driven by an ultrasound source in order to stimulate the samples in a relatively large frequency range. Three transducers, denoted as $A, B$, and $C$ respectively, were used. The nominal power of transducer $\mathrm{A}, \mathrm{B}$, and $\mathrm{C}$ is $100 \mathrm{~W}, 100 \mathrm{~W}$, and $50 \mathrm{~W}$ respectively.

The reference sample is an adhesive bonded composite plate which consists of an aluminium alloy plate and a cork plate, as shown in Fig.2. The thickness of the cork plate is $2 \mathrm{~mm}$. Eight premade disbonding defects were created by drilling flat bottom holes with a depth of $0.1 \mathrm{~mm}$ and diameters of $15 \mathrm{~mm}, 8 \mathrm{~mm}, 5 \mathrm{~mm}, 10 \mathrm{~mm}$ on the aluminium alloy plate before bonding, as shown in Fig.2(a). The fixture with a clamping device are shown in Fig.3. A plastic gasket was adopted to couple the ultrasonic wave into the sample.

Set the excitation duration to 10 seconds, the frame rate to $1 \mathrm{~Hz}$, the number of background frames before the start of excitation to 5 , the total number of frames to 60 , then an image sequence of 60 frames was recorded in each time The excess temperature images were obtained by subtracting the ambient temperature image from subsequent images in the analyzed sequence.

The excess temperature images at the end of excitation ( $t=10 \mathrm{~s})$ using transducer $\mathrm{A}, \mathrm{B}$ and $\mathrm{C}$ under their resonance frequencies are shown in Fig. 4. The temperature difference evolutions over the four defects in the upper row under the $21.5 \mathrm{kHz}$ excitation by transducer A are shown in Fig.5. The temperature difference over a defect was obtained by subtracting an average reference temperature over a non-defect area from the average temperature over the defect. The defects in Fig.4 (a) \& (b) are obvious, and the thermal mode at the non-defect areas is also clear. The thermal mode phenomenon cannot be explained by the conventional viewpoint of friction heat. 


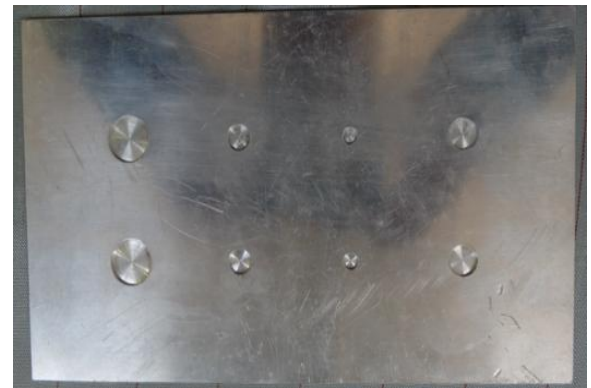

(a) Photo of the aluminium alloy plate

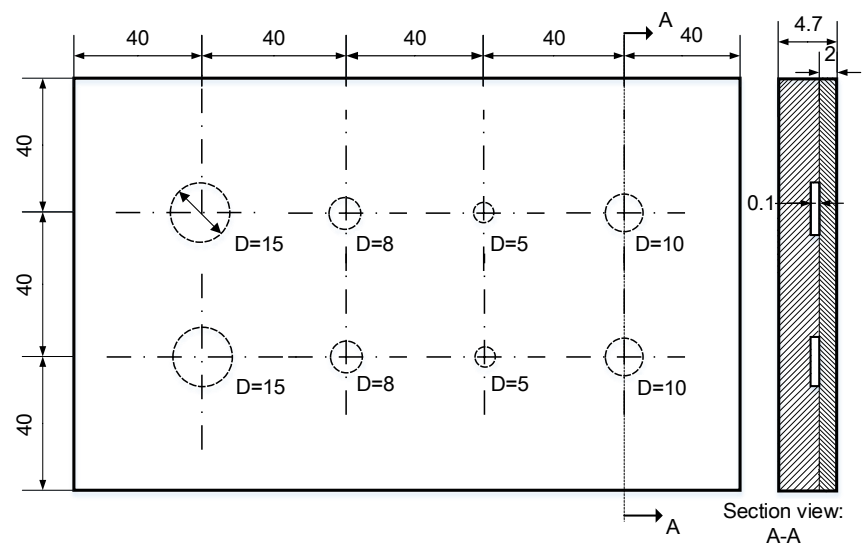

(b) drawing of the composite plate (unit: $\mathrm{mm}$ )

Fig.2. The sample

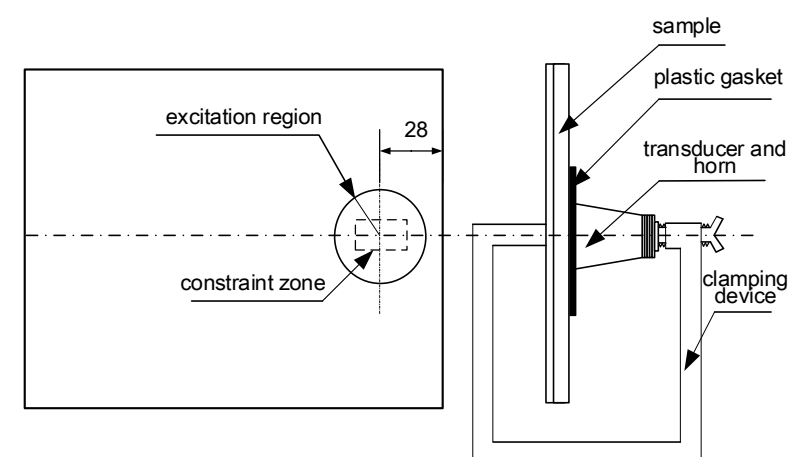

Fig.3. Schematic diagram of the fixture with a clamping device

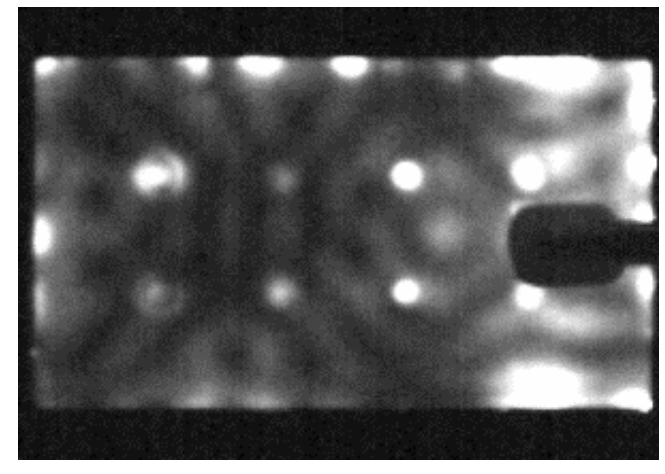

(a) Transducer $A, f=21.5 \mathrm{kHz}$

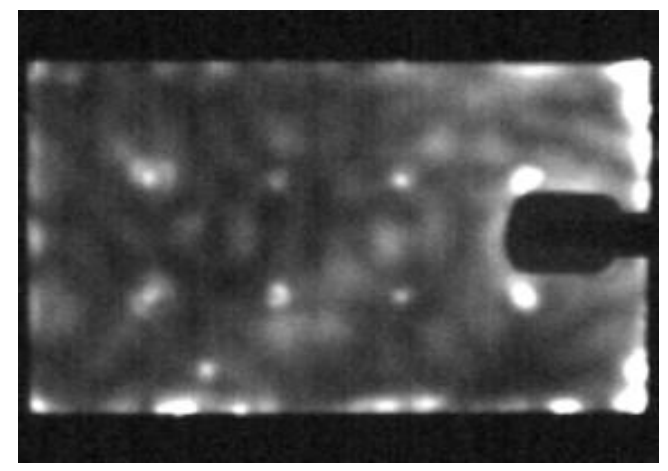

(b) Transducer $B, f=26.5 \mathrm{kHz}$

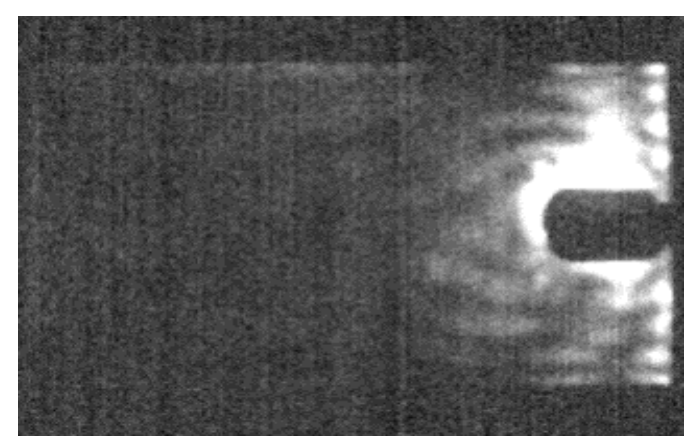

(c) Transducer $C, f=39 \mathrm{kHz}$

Fig.4. Excess temperature images at $t=10$ s using different transducers under their resonance frequencies (f) 


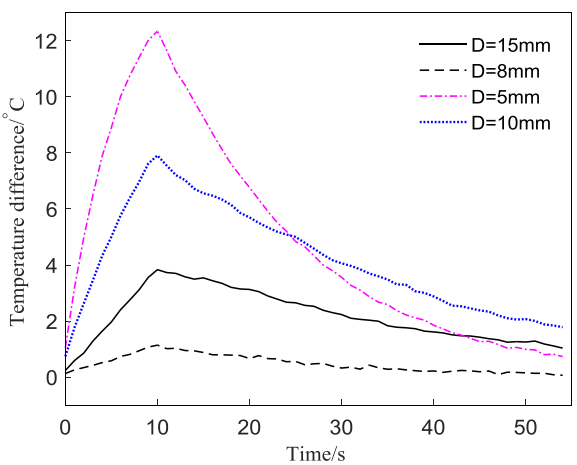

Fig.5. Temperature difference evolutions over the four defects in the upper row under the $21.5 \mathrm{kHz}$ excitation by transducer $A$ ( $D$ denotes the diameter of the defect)

All the eight defects can be seen under the $21.5 \mathrm{kHz}$ and $26.5 \mathrm{kHz}$ excitation, but most of the defects cannot be seen under the $39 \mathrm{kHz}$ excitation, different excitation frequency has different thermal effect to a certain defect. In other words, the excitation frequency has 'selective' heating to defects. This may not be simply attributed to that the output power is different at the resonance frequency of each transducer. To understand the relationship between the heat generation at defects and the excitation frequency, the numerical simulation was conducted by using finite element method.

\section{Numerical simulation}

A thermo-mechanical coupled field model based on viscoelasticity heat was established according to section 2 . The related boundary and initial condition were set as the followings.

(1) On the place contacted with the transducer, the amplitude of the base displacement excitation was $2 \mu \mathrm{m}$, i.e.

$$
W_{\mathrm{b}}(t)=2 \times 10^{-6} \sin (2 \pi f t)
$$

(2) The initial condition of the heat conduction was

$$
\left.T\right|_{t=0}=298.15 \mathrm{~K}
$$

(3) The convective boundary condition on the outer surfaces of the specimen was as follows.

$$
k \nabla T=h_{r}\left(T_{0}-T\right)
$$

where $T_{0}$ is the ambient temperature, $h_{r}$ is the convective heat transfer coefficient on the outer surfaces. The convective heat transfer coefficient of the aluminium alloy and the cork were regarded as equal,

$$
h_{r}=5 \mathrm{~W} /\left(\mathrm{m}^{2} \cdot \mathrm{k}\right)
$$

(4) At the interface $\left(z=Z_{0}\right)$ between the cork layer and the aluminium alloy layer, the temperature is continuous, and it satisfies Eq.(10).

$$
\left.T_{1}\right|_{z=Z_{0}}=\left.T_{2}\right|_{z=Z_{0}}
$$

The material properties were taken as that in Table 1.

Table 1. Material properties

\begin{tabular}{|c|c|c|c|c|c|c|}
\hline Material & $\begin{array}{c}\text { Young's } \\
\text { modulus } \\
E(\mathrm{~Pa})\end{array}$ & $\begin{array}{c}\text { Poisson's } \\
\text { ratio } \\
\mu\end{array}$ & $\begin{array}{c}\text { Density } \\
\rho\left(\mathrm{kg} \cdot \mathrm{m}^{-3}\right)\end{array}$ & $\begin{array}{c}\text { Loss factor } \\
\eta[10]\end{array}$ & $\begin{array}{c}\text { Specific heat } \\
c\left(\mathrm{~J} \cdot \mathrm{kg}^{-1} \mathrm{~K}^{-1}\right)\end{array}$ & $\begin{array}{c}\text { Thermal } \\
\text { conductivity } \\
\mathrm{k}\left(\mathrm{W} \cdot \mathrm{m}^{-1} \mathrm{~K}^{-1}\right)\end{array}$ \\
\hline Aluminium alloy & $7.0 \times 10^{10}$ & 0.33 & 2696 & $5 \times 10^{-5}$ & 900 & 138 \\
\hline Cork & $3.92 \times 10^{8}$ & 0.46 & 470 & 0.01 & 1790 & 0.08 \\
\hline Air & $6 \times 10^{-3}$ & 0 & 1.174 & 0 & 1013 & 0.02624 \\
\hline
\end{tabular}

Set the excitation frequency $f$ to $21.5 \mathrm{kHz}$, the simulation excess temperature response on the surface was calculated, as shown in Fig.6. Set the excitation frequency $f$ to $39 \mathrm{kHz}$, the temperature image at time $10 \mathrm{~s}$ is shown in Fig.7. The theoretical results are comparable to the experimental results in Fig.4 and Fig.5. 


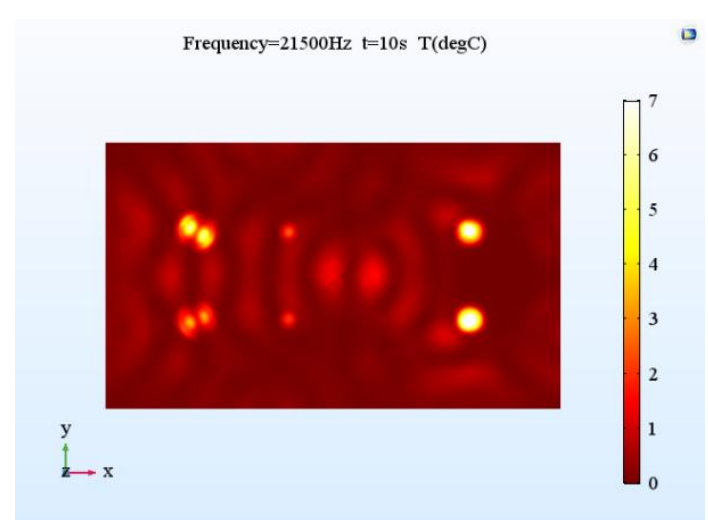

(a)

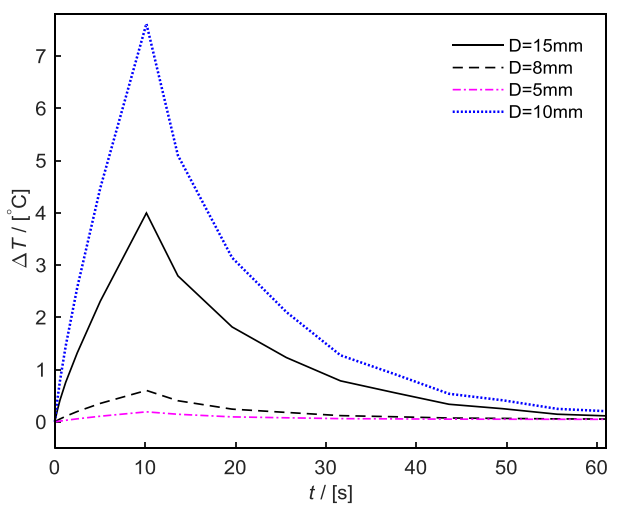

(b)

Fig.6 The simulation excess temperature response on the surface: (a) the excess temperature image at time 10s under $21.5 \mathrm{kHz}$ excitation; (b) the temperature difference evolutions of the four defect areas in the upper row (D denotes the diameter of the defect)

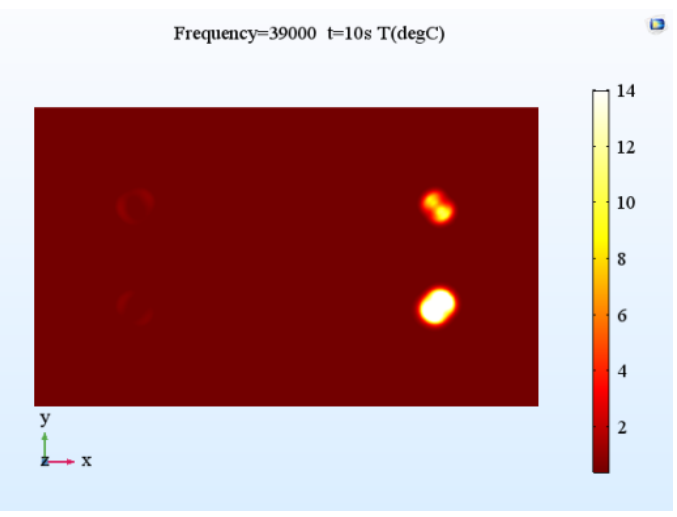

Fig.7. The excess temperature image at time 10 s under $39 \mathrm{kHz}$ excitation

By conducting the modal analysis of the whole plate, it is found that a local effect is obvious over the defects in some higher order mode shapes. Fig.8 shows the mode shapes with significant local effect over defects. We call the corresponding mode frequency as higher mode characteristic frequency. For a specific defect area, this mode frequency has different orders just like the natural frequency. In this paper, we only focus the first order higher mode characteristic frequency.

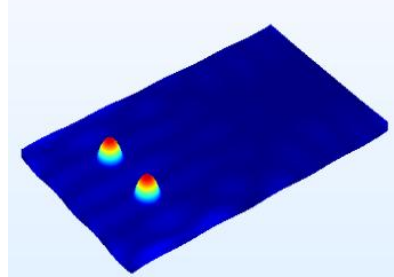

(a) 48th order, $12938 \mathrm{~Hz}$

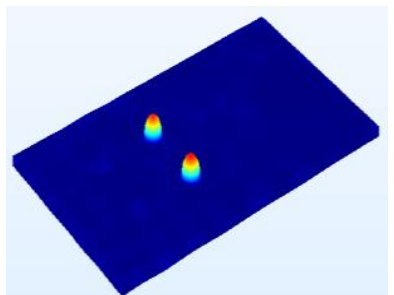

(b) 124th order, $33813 \mathrm{~Hz}$

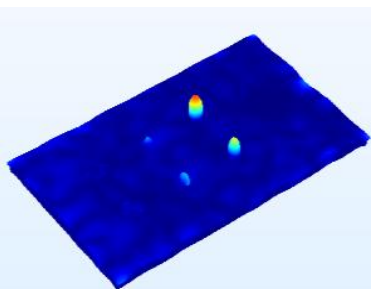

(c) 281 th order, $61029 \mathrm{~Hz}$

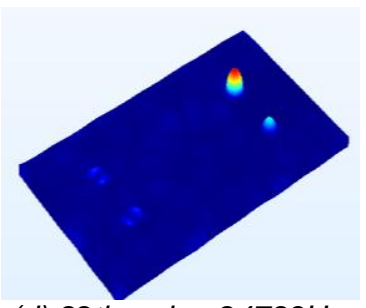

(d) 82 th order, $24783 \mathrm{~Hz}$

Fig.8. The mode shapes with significant local effect at defects and the higher mode characteristic frequencies

The LDR frequencies for a circular cork plate under clamped and simple supported boundary conditions are shown in table 2. Mostly the first order frequency under the clamped boundary condition is used.

To compare the thermal effect of higher mode characteristic frequencies and the LDR frequencies, the coupled field simulations at single frequency excitation were conducted. The simulation excess temperature images are shown in Fig. 8 and Fig.9. 
Table 2. $L D R$ frequencies $(\mathrm{Hz})$ of circular cork plates with a thickness of $2 \mathrm{~mm}$

\begin{tabular}{|c|l|l|l|l|l|}
\hline \multirow{2}{*}{ Boundary Condition } & \multirow{2}{*}{ Order } & \multicolumn{4}{|c|}{ Diameter $(\mathrm{mm})$} \\
\cline { 3 - 6 } & & 15 & 8 & 5 & 10 \\
\hline \multirow{2}{*}{ Clamped } & 1 st & 14563 & 38218 & 69997 & 27715 \\
\cline { 2 - 6 } & 2nd & 26470 & 63153 & 110707 & 47264 \\
\hline \multirow{2}{*}{ Simple Supported } & 1 st & 7864.4 & 20947 & 33101 & 15388 \\
\cline { 2 - 6 } & 2nd & 18602 & 37844 & 47206 & 31235 \\
\hline
\end{tabular}

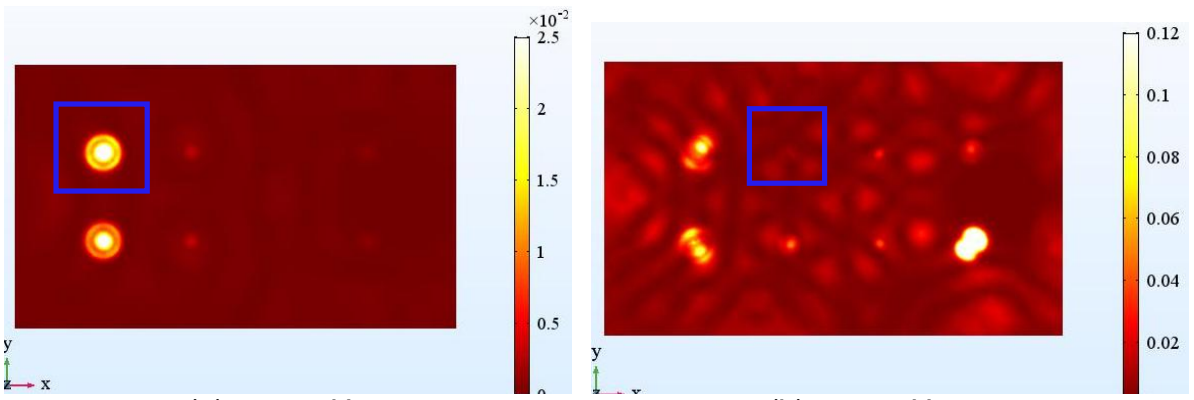

(a) $14563 \mathrm{~Hz}$

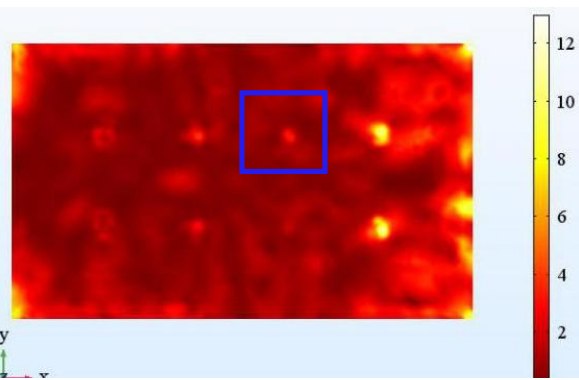

(c) $69997 \mathrm{~Hz}$ (b) $38218 \mathrm{~Hz}$

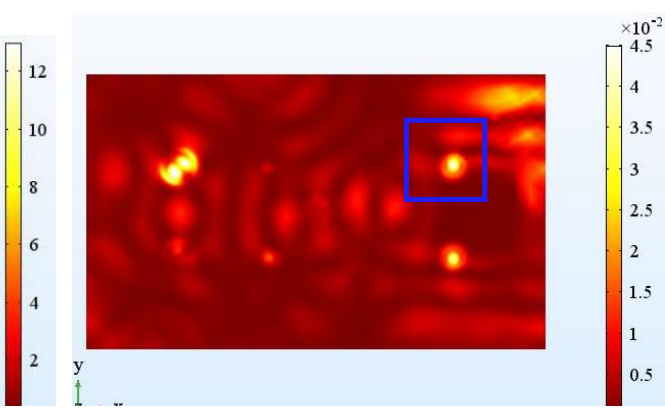

(d) $27715 \mathrm{~Hz}$

Fig.8. Simulation excess temperature image map at time $t=10$ s under the $L D R$ frequencies excitation

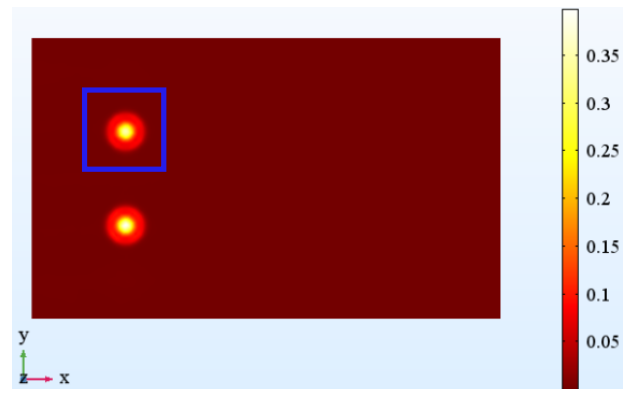

(a) $12938 \mathrm{~Hz}$

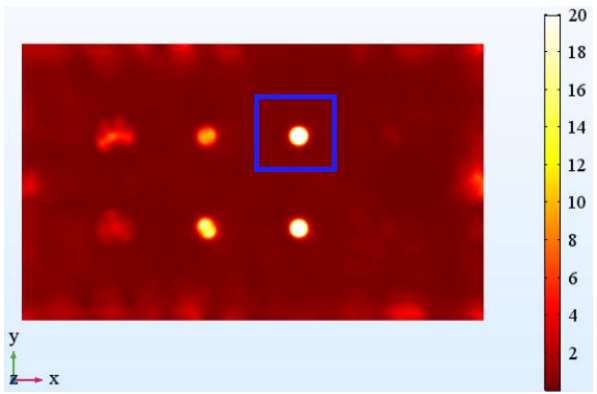

(c) $61029 \mathrm{~Hz}$

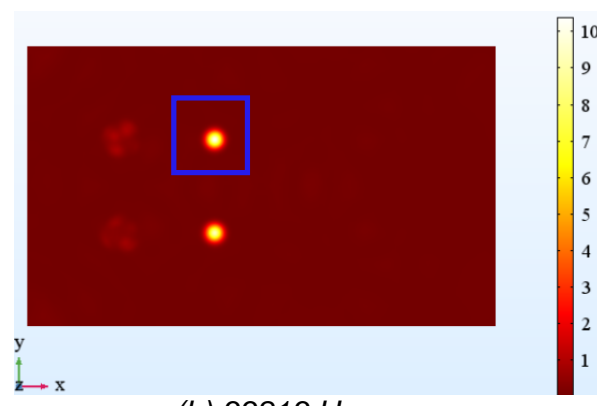

(b) $33813 \mathrm{~Hz}$

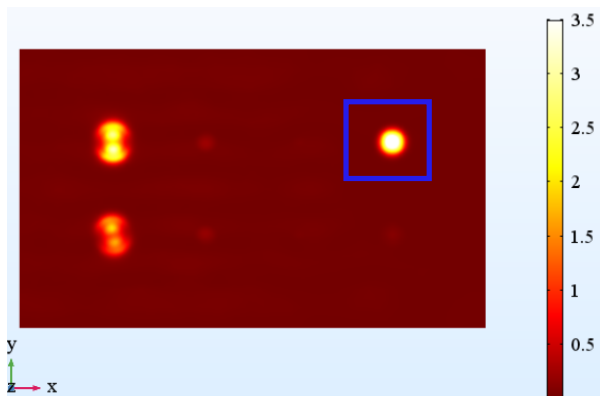

(d) $24783 \mathrm{~Hz}$

Fig.9. Simulation excess temperature image at time $t=10$ s under the higher mode characteristic frequencies excitation 
As we can see in Fig.8 and Fig.9, excitation using the higher mode characteristic frequencies has better thermal effect than using the LDR frequencies. Although some targeted defects are obvious in Fig.8, the excess temperatures over the defects are too small except for Fig.8(c). So the LDR frequency is not an exact indication for the optimal excitation frequency, and it should be replaced by the higher mode characteristic frequency.

The higher mode characteristic frequency is between the LDR frequency under simple supported boundary condition and the LDR frequency under clamped boundary condition. As can be seen in table 2 and Fig.7, all the four first order LDR frequencies are slightly larger than the corresponding higher mode characteristic frequency. In fact, the aluminium layer is not an ideal rigid body, so regarding the boundary of the debonding part of the cork as the clamped boundary will increase the mode frequency, whereas regarding the boundary as the simple supported boundary will decrease the mode frequency. In spite of the worse thermal effect of the LDR frequency, the LDR theory provides a simple and quick way to determine the optimal excitation frequency range in which the higher mode characteristic frequency locates.

\section{Conclusions}

(1) The viscoelastic loss of the non-metallic material in the composite plate is the leading factor of the local temperature increase in disbonding areas during VT.

(2) A disbonding defect has its optimal excitation frequency. Conversely, an excitation frequency has the 'selective' effect on its garget defect. For an unknown defect, in order to obtain a better detection, the excitation frequency should be adjustable in a large range, or the multiple frequency excitations should be employed.

(3) The higher mode characteristic frequency is a more exact indication for the optimal excitation frequency than the LDR frequency. The higher mode characteristic frequency is between the LDR frequency under the simple supported boundary condition and the LDR frequency under the clamped boundary condition.

\section{Acknowledgment}

This work was supported by the National Natural Science Foundation of China (61571028, U1433122).

\section{REFERENCES}

[1] Renshaw J, Chen J C, Holland S D, Thompson R B. The sources of heat generation in vibrothermography. NDE \& $E$ International, 2011; 44(8): 736-739.

[2] Han X, Islam M, Newaz G. Finite element modeling of the heating of cracks during sonic infrared imaging Journal of Applied Physics, 2006; 99(7): 1-7.

[3] Renshaw J, Holland S D, Barnard D J. Viscous material-filled synthetic defects for vibrothermography. NDE \& E International, 2009; 42: 753-756.

[4] Rizi A S, Hedayatrasa S, Maldague X, Vukhanh T. FEM modeling of ultrasonic vibrothermography of a damaged plate and qualitative study of heating mechanisms. Infrared Physics \& Technology, 2013; 61: 101-110.

[5] Mabrouki F, Thomas M, Genest M, Fahr A. Frictional heating model for efficient use of vibrothermography. NDT\&E International, 2009; 42: 345-352.

[6] Montanini R, Freni F. Correlation between vibrational mode shapes and viscoelastic heat generation in vibrothermography. NDE \& E International, 2013; 58: 43-48.

[7] Solodov I, Rahammer M, Derusova D, Busse G. Highly-efficient and noncontact vibro-thermography via local defect resonance. Quantitative Infrared Thermography Journal, 2015; 12(1): 98-111.

[8] Solodov I, Bai J, Bekgulyan S, Busse G. A local defect resonance to enhance acoustic wave-defect interaction in ultrasonic non-destructive evaluation, Applied Physics Letters, 2011; 99: 211911(1-4). doi: $10.1063 / 1.3663872$

[9] Miller J K, Woods D C, Rhoads J F. Thermal and mechanical response of particulate composite plates under inertial excitation. Journal of Applied Physics, 2014; 116: 244904(1-10).

[10] Chang G. Viscoelastic damping materials. Beijing: National Defense Industry Press, 2012, 9-38 